Portland State University

PDXScholar

Electrical and Computer Engineering Faculty

Publications and Presentations

4-1-2007

\title{
Spontaneous Mode Locking in Mixed-Broadened Laser Oscillators
}

Pitak Chenkosol

Portland State University

Lee W. Casperson

Portland State University

Follow this and additional works at: https://pdxscholar.library.pdx.edu/ece_fac

Part of the Electrical and Computer Engineering Commons

Let us know how access to this document benefits you.

Citation Details

Pitak Chenkosol and Lee W. Casperson, "Spontaneous mode locking in mixed-broadened laser oscillators," J. Opt. Soc. Am. B 24, 1199-1210 (2007).

This Article is brought to you for free and open access. It has been accepted for inclusion in Electrical and Computer Engineering Faculty Publications and Presentations by an authorized administrator of PDXScholar. Please contact us if we can make this document more accessible: pdxscholar@pdx.edu. 


\title{
Spontaneous mode locking in mixed-broadened laser oscillators
}

\author{
Pitak Chenkosol* and Lee W. Casperson ${ }^{\dagger}$ \\ Department of Electrical and Computer Engineering and Department of Physics, Portland State University, \\ P.O. Box 751, Portland, Oregon 97207-0751, USA
}

Received February 28, 2006; revised December 6, 2006; accepted December 29, 2006; posted January 18, 2007 (Doc. ID 68265); published April 17, 2007

\begin{abstract}
A theoretical model is reported for spontaneous mode locking in mixed-broadened laser oscillators. Experimental observations of this effect have been available for many years, but no rigorous interpretation has been given. Numerical calculations emphasize the case of a high-gain xenon laser, for which extensive experimental data have been published. Complex pulsation characteristics are observed as the cavity length and pumping rate are varied, and the theoretical results are in good agreement with the experimental data. (C) 2007 Optical Society of America

OCIS codes: $270.3430,270.3100,020.1670,140.3460$.
\end{abstract}

\section{INTRODUCTION}

The most basic prerequisite for mode locking in any system is the coexistence of several distinguishable modes. Laser mode locking usually refers to the circumstance in which the fields associated with the longitudinal modes of a laser cavity superpose to form one or more pulses circulating periodically (or sometimes chaotically) around the laser. In the simplest cases, these modes have constant amplitude, are equally spaced in frequency, and have constant phase differences. A review of early mode locking studies, especially those relating to spontaneous mode locking, has been given by Tarroja et al. ${ }^{1}$ These studies have included both theory and experiment.

Although many self-mode-locking mechanisms involve only longitudinal modes, self-locking of transverse modes has also been reported. However, with the inclusion of an appropriate aperture in the laser cavity, one can ensure that a laser operates in one transverse mode and that only longitudinal self-locking can occur. In this case the cavity length plays a major role in determining the nature of the self-locking. When the cavity length is not much greater than that of the gain medium, mode-pulling effects due to the dispersion in the medium may cause unequally spaced mode frequencies. The regular self-locking output pulsations that nevertheless are sometimes observed in these laser systems are made possible by more complex nonlinear interactions between the electric field and the gain medium.

Some lasers can undergo transitions from regular periodic mode locking to partial locking and irregular pulsations. These transitions have been the subject of many theoretical investigations. Risken and Nummedal studied the conditions for multimode laser instability in homogeneously broadened two-level unidirectional ring lasers. ${ }^{2}$ It may be noted that in the simplest models for saturating homogeneously broadened lasers only the cavity mode that is closest to the line center of the atomic resonance has net gain. The observed multimode phenomena in many of these lasers appear with the onset of instability. On the other hand, inhomogeneously broadened lasers may intrinsically have multiple cavity modes with enough gain to oscillate, and in these lasers time-dependent intensity signals are inevitable. Instabilities can also be induced by adding to the simplest models some additional nonlinear physical process such as saturable absorption or Kerr lensing. It has sometimes been difficult to achieve a convincing experimental demonstration that corresponds closely to a laser instability that has been postulated theoretically, and similarly it is not always straightforward to identify the correct theoretical interpretation of an experimentally observed instability.

Many plane-wave studies of dynamical phenomena in lasers have been reported over the years. However, the number of possible interpretations of spontaneous pulsations in the simplest lasers such as we are considering, and the number of successful simulations of such pulsations have been limited. For example, it was suggested that the postulated coherent Lorenz-Haken instability of a single-mode homogeneously broadened laser ${ }^{3}$ had been observed in a far-infrared system, but that interpretation has been questioned. ${ }^{4}$ Similarly, it was argued that the multimode homogeneously broadened Risken-Nummedal laser instability had also been observed. But it has recently been stated that "no experimental demonstration of the type of instability described by Risken and Nummedal, and by Graham and Haken, has ever been reported." Thus, there is value and interest in clarifying the relationship between theory and experiment for any physically simple spontaneously pulsing laser oscillator.

The focus in this study has been on developing the first rigorous theoretical interpretation of well-known spontaneous pulsations in multimode inhomogeneously broadened (or mixed-broadened) laser oscillators. This basic instability has been known experimentally in low-pressure gas lasers since $1970,{ }^{6,7}$ but it has not been modeled previously. The model reported here starts from the semiclas- 
sical laser equations and is developed in Section 2. The application of this model including normalization to the laser threshold and establishing initial conditions is described in Section 3. The numerical solutions emphasized here focus on high-gain xenon lasers for which spontaneous mode locking has long been known ${ }^{6,7}$ and for which detailed experimental data are available. ${ }^{1}$ Cavity lengths up to $66 \mathrm{~m}$ have been considered, and as discussed in Section 4 the theoretical results are in good agreement with experimental data.

\section{GENERAL MODEL}

The starting point for this analysis is a set of MaxwellSchrödinger laser equations that is identical to that used in a previous study of spontaneous pulsations in singlemode mixed-broadened ring laser oscillators. ${ }^{8}$ Beginning with that model, the normalized equations for the time

and space dependences of the field amplitude and the polarization and population elements of the laser medium for such a laser can be written:

$$
\begin{aligned}
\frac{\partial A(z, t)}{\partial t}+v_{p} \frac{\partial A(z, t)}{\partial z}= & -\frac{\sigma}{2 \varepsilon_{1}}\left[\left\{1-i \delta\left(y-y_{0}\right)\right\} A(z, t)\right. \\
& \left.-i \int_{-\infty}^{\infty} \int_{-\infty}^{\infty} P(V, U, z, t) \mathrm{d} V \mathrm{~d} U\right],
\end{aligned}
$$

$$
\begin{aligned}
\frac{\partial P(V, U, z, t)}{\partial t}+\varepsilon u V \frac{\partial P(V, U, z, t)}{\partial z} \\
=-\gamma[\{1-i(y-U-V)\} P(V, U, z, t) \\
\quad+i A(z, t) D(V, U, z, t)],
\end{aligned}
$$

where $A$ represents the complex amplitude of the electric field, $D$ is the population difference between the upper and lower states of the laser transition, and $M$ is the population sum.

The other variables in Eqs. (1)-(4) include the normalized velocity of the atoms or molecules,

$$
V=\frac{v}{\varepsilon u}=\frac{k v}{\gamma},
$$

the normalized intrinsic frequency of the transition for a particular class $\alpha$ of atoms,

$$
U=\frac{\omega_{\alpha}-\omega_{0}}{\gamma},
$$

$u$ as the most probable speed of the atoms, $k$ as Boltzmann's constant, $\gamma$ as the polarization decay rate, $\omega_{0}$ as a reference frequency for the laser transition, and $\varepsilon$ as

$$
\left(\Delta \nu_{h} / \Delta \nu_{D}\right)(\ln 2)^{1 / 2}
$$

the natural damping ratio. ${ }^{9}$ Other parameters include the phase velocity $v_{p}$, the conductivity of the laser medium $\sigma$, the background permittivity of the laser medium $\varepsilon_{1}$, the normalized lasing frequency $y=\left(\omega-\omega_{0}\right) / \gamma$, the normalized cavity frequency $y_{0}=\left(\Omega-\omega_{0}\right) / \gamma$, the polarization decay rate $\gamma$, the pump rates $\lambda_{d}$ and $\lambda_{m}$, the decay rate ratios $\gamma_{1}$ and $h_{1}$ to $h_{4}$, and the velocity-changing collision kernels $\Gamma_{a}$ and $\Gamma_{b}$. Further information on the derivation of these equations and the significance of the various parameters is given in Ref. 8, and the decay ratios are defined in Ref. 10.

The model summarized in Eqs. (1)-(4) can be applied to single-mode instabilities as it was in Ref. 8, but it is also valid for arbitrary multimode phenomena. In Ref. 1 an equivalent model was applied to spontaneous mode locking under the constraint that the pulsations be exactly periodic. Experiments show that the pulsations are not always periodic, and the purpose of the present study is to 
obtain more general solutions of the model for comparison with experimental data.

Equations (1)-(4) allow for the possibility of a distribution of the natural transition center frequencies ( $U$ in the normalized units). However, as in Ref. 8, the polarization and the population variables are regarded as delta functions of $U$, and Eqs. (1)-(4) reduce to the following set:

$$
\begin{gathered}
\frac{\partial A(z, t)}{\partial t}+v_{p} \frac{\partial A(z, t)}{\partial z}=-\frac{\sigma}{2 \varepsilon_{1}}\left[\left\{1-i \delta\left(y-y_{0}\right)\right\} A(z, t)\right. \\
\left.-i \int_{-\infty}^{\infty} P(V, z, t) \mathrm{d} V\right], \\
\frac{\partial P(V, z, t)}{\partial t}+\varepsilon u V \frac{\partial P(V, z, t)}{\partial z}=-\gamma[\{1-i(y-V)\} P(V, z, t) \\
\left.\frac{\partial D(V, z, t)}{\partial t}+\varepsilon u V \frac{\partial D(V, z, t)}{\partial z}+i A(z, t) D(V, z, t)\right], \\
=\lambda_{d}(V, z, t)-h_{1} D(V, z, t)-h_{2} M(V, z, t) \\
+i \gamma_{1}\left[A(z, t) P^{*}(V, z, t)-A^{*}(z, t) P(V, z, t)\right] \\
\quad+\frac{\varepsilon \Gamma_{a}}{2 \sqrt{\pi}} \exp \left(-\varepsilon^{2} V^{2}\right) \int_{-\infty}^{\infty}\left[M\left(V^{\prime}, z, t\right)+D\left(V^{\prime}, z, t\right)\right] \mathrm{d} V^{\prime} \\
\quad-\frac{\varepsilon \Gamma_{b}}{2 \sqrt{\pi}} \exp \left(-\varepsilon^{2} V^{2}\right) \int_{-\infty}^{\infty}\left[M\left(V^{\prime}, z, t\right)-D\left(V^{\prime}, z, t\right)\right] \mathrm{d} V^{\prime},
\end{gathered}
$$

$$
\begin{aligned}
& \frac{\partial M(V, z, t)}{\partial t}+\varepsilon u V \frac{\partial M(v, z, t)}{\partial z} \\
& =\lambda_{m}(V, z, t)-h_{3} M(V, z, t)-h_{4} D(V, z, t) \\
& \quad+\frac{\varepsilon \Gamma_{a}}{2 \sqrt{\pi}} \exp \left(-\varepsilon^{2} V^{2}\right) \int_{-\infty}^{\infty}\left[M\left(V^{\prime}, z, t\right)+D\left(V^{\prime}, z, t\right)\right] \mathrm{d} V^{\prime} \\
& \quad+\frac{\varepsilon \Gamma_{b}}{2 \sqrt{\pi}} \exp \left(-\varepsilon^{2} V^{2}\right) \int_{-\infty}^{\infty}\left[M\left(V^{\prime}, z, t\right)-D\left(V^{\prime}, z, t\right)\right] \mathrm{d} V^{\prime}
\end{aligned}
$$

Equations (8)-(11) can be solved subject to the ringlaser boundary condition

$$
A(0, t)=\sqrt{R} A\left(l, t-\frac{L-l}{v_{p}}\right),
$$

where $l$ is the length of the gain medium, $L$ is the total length of the cavity, and $R$ is the output mirror reflectivity. We assume that all other mirrors that are used to form the ring cavity are ideal reflectors. We focus in this study on the situation in which the output mirror reflectivity is high, i.e., $R \approx 1$. The total round trip losses of the electric field can then combined and uniformly distributed along the resonator length. In addition, as in Ref. 1, we introduce a new set of independent variables:

$$
\begin{gathered}
\eta=z \\
\tau=t+\left(\frac{L-l}{v_{p}}\right) \frac{z}{l}
\end{gathered}
$$

The transformations in Eqs. (13) and (14) eliminate the time delay in the boundary condition given by Eq. (12) in the new reference frame, so that this boundary condition can be written as

$$
A(0, \tau)=A(l, \tau) .
$$

In terms of the variables given in Eqs. (13) and (14), one can write

$$
\begin{gathered}
\frac{\partial A(z, t)}{\partial t}=\frac{\partial A(\eta, \tau)}{\partial \eta} \frac{\partial \eta}{\partial t}+\frac{\partial A(\eta, \tau)}{\partial \tau} \frac{\partial \tau}{\partial t}=\frac{\partial A(\eta, \tau)}{\partial \tau}, \\
\frac{\partial A(z, t)}{\partial z}=\frac{\partial A(\eta, \tau)}{\partial \eta} \frac{\partial \eta}{\partial z}+\frac{\partial A(\eta, \tau)}{\partial \tau} \frac{\partial \tau}{\partial z} \\
=\frac{\partial A(\eta, \tau)}{\partial \eta}+\frac{\partial A(\eta, \tau)}{\partial \tau}\left(\frac{1}{v_{p}} \frac{L}{l}-\frac{1}{v_{p}}\right) .
\end{gathered}
$$

With these derivative transformations, the left-hand side of Eq. (8) can be written

$$
\begin{aligned}
\frac{\partial A(z, t)}{\partial t}+v_{p} \frac{\partial A(z, t)}{\partial z}= & \frac{\partial A(\eta, \tau)}{\partial \tau} \\
& +v_{p}\left[\frac{\partial A(\eta, \tau)}{\partial \eta}+\frac{\partial A(\eta, \tau)}{\partial \tau}\left(\frac{1}{v_{p}} \frac{L}{l}-\frac{1}{v_{p}}\right)\right] \\
= & \frac{L}{l} \frac{\partial A(\eta, \tau)}{\partial \tau}+v_{p} \frac{\partial A(\eta, \tau)}{\partial \eta} .
\end{aligned}
$$

Thus, Eq. (8) becomes

$$
\begin{aligned}
\frac{\partial A(\eta, \tau)}{\partial \tau}+v_{p} \frac{l}{L} \frac{\partial A(\eta, \tau)}{\partial \eta}= & -\gamma_{c} \frac{l}{L}\left[\left\{1-i \delta\left(y-y_{0}\right)\right\} A(\eta, \tau)\right. \\
& \left.-i \int_{-\infty}^{\infty} P(V, \eta, \tau) \mathrm{d} V\right]
\end{aligned}
$$

where

$$
\gamma_{c}=\frac{1}{2 t_{c}}=\frac{\sigma}{2 \varepsilon_{1}}
$$

is the total electric field decay rate, and $t_{c}$ is the total cavity lifetime.

In similar fashion one can rewrite Eqs. (9)-(11) as, respectively,

$$
\begin{aligned}
& \frac{\partial P(V, \eta, \tau)}{\partial \tau}+\varepsilon u V \chi(V) \frac{\partial P(V, \eta, \tau)}{\partial \eta} \\
& \quad=-\gamma \chi(V)[\{1-i(y-V)\} P(V, \eta, \tau)+i A(\eta, \tau) D(V, \eta, \tau)],
\end{aligned}
$$




$$
\begin{aligned}
& \frac{\partial D(V, \eta, \tau)}{\partial \tau}+\varepsilon u V \chi(V) \frac{\partial D(V, \eta, \tau)}{\partial \eta} \\
& =\chi(V)\left[\lambda_{d}(V, \eta, \tau)-h_{1} D(V, \eta, \tau)-h_{2} M(V, \eta, \tau)\right. \\
& \quad+i \gamma_{1}\left\{A(\eta, \tau) P^{*}(V, \eta, \tau)-A^{*}(\eta, \tau) P(V, \eta, \tau)\right\} \\
& +\frac{\varepsilon \Gamma_{a}}{2 \sqrt{\pi}} \exp \left(-\varepsilon^{2} V^{2}\right) \int_{-\infty}^{\infty}\left\{M\left(V^{\prime}, \eta, \tau\right)+D\left(V^{\prime}, \eta, \tau\right)\right\} \mathrm{d} V^{\prime} \\
& \left.\quad-\frac{\varepsilon \Gamma_{b}}{2 \sqrt{\pi}} \exp \left(-\varepsilon^{2} V^{2}\right) \int_{-\infty}^{\infty}\left\{M\left(V^{\prime}, \eta, \tau\right)-D\left(V^{\prime}, \eta, \tau\right)\right\} \mathrm{d} V^{\prime}\right],
\end{aligned}
$$

$$
\begin{aligned}
& \frac{\partial M(V, \eta, \tau)}{\partial \tau}+\varepsilon u V \chi(V) \frac{\partial M(V, \eta, \tau)}{\partial \eta} \\
& =\chi(V)\left[\lambda_{m}(V, \eta, \tau)-h_{3} M(V, \eta, \tau)-h_{4} D(V, \eta, \tau)\right. \\
& +\frac{\varepsilon \Gamma_{a}}{2 \sqrt{\pi}} \exp \left(-\varepsilon^{2} V^{2}\right) \int_{-\infty}^{\infty}\left\{M\left(V^{\prime}, \eta, \tau\right)+D\left(V^{\prime}, \eta, \tau\right)\right\} \mathrm{d} V^{\prime} \\
& \left.+\frac{\varepsilon \Gamma_{b}}{2 \sqrt{\pi}} \exp \left(-\varepsilon^{2} V^{2}\right) \int_{-\infty}^{\infty}\left\{M\left(V^{\prime}, \eta, \tau\right)-D\left(V^{\prime}, \eta, \tau\right)\right\} \mathrm{d} V^{\prime}\right],
\end{aligned}
$$

where

$$
\chi(V)=\left[1+\frac{\varepsilon u V}{v_{p}}\left(\frac{L}{l}-1\right)\right]^{-1}
$$

Equations (20) and (22)-(24) form a general semiclassical laser model that can be used to study the dynamical behavior of mixed-broadened unidirectional ring laser oscillators with uniform distributed losses. This set of laser equations will be solved subject to the boundary condition given in Eq. (15).

\section{THRESHOLD PARAMETER AND INITIAL CONDITIONS}

In order to obtain meaningful solutions to the dynamical equations, it is helpful if the various quantities involved can be expressed in term of a threshold parameter that relates the actual laser pump rates to their values when the laser is at the lasing threshold. This threshold parameter, in addition, provides a direct link between the experiments and numerical computations.

Consider a laser that operates at the lasing threshold and line-center tuning $\left(y=y_{0}=0\right)$. For this case, one can write steady-state laser equations for Eqs. (20) and (22) as

$$
0=-A_{s, t h}+i \int_{-\infty}^{\infty} P_{s, t h}(V) \mathrm{d} V
$$

$$
\begin{gathered}
0=-\left(A_{r s, t h}+i A_{i s, t h}\right)+i \int_{-\infty}^{\infty}\left[P_{r s, t h}(V)+i P_{i s, t h}(V)\right] \mathrm{d} V \\
0=(1+i V) P_{s, t h}(V)+i A_{s, t h} D_{s, t h}(V), \\
0=(1+i V)\left[P_{r s, t h}(V)+i P_{i s, t h}(V)\right]+i\left(A_{r s, t h}+i A_{i s, t h}\right) D_{s, t h}(V),
\end{gathered}
$$

where $A_{r s, t h}, P_{r s, t h}(V)$ and $A_{i s, t h}, P_{i s, t h}(V)$ are the real and imaginary parts of the steady-state electric field $A_{s, t h}$ and the polarization $P_{s, t h}(V)$, respectively.

By line-center tuning is meant that both the nondispersed cavity frequency and the actual oscillation frequency (for single-mode oscillation near threshold) are coincident with the center frequency of the mixedbroadened gain distribution. Thus, threshold will be defined here by the condition that a single infinitesimalamplitude cavity mode exactly at line center has a gain exactly equal to its loss. It should be emphasized that this restriction to line-center tuning is employed only for the purpose of defining the laser threshold in terms of other parameters of the model. For all other dynamical calculations, the fields are computed for a given length cavity without any $a$ priori assumptions about frequency tuning. Thus, a laser is said to be mode-locked when the total electric field is in the form of pulses that are approximately periodic in time with a period that is equal either to the round-trip time or to a submultiple of the roundtrip time. In the frequency domain such a field would be represented by a number of approximately equally spaced frequency components that may be referred to as longitudinal cavity modes and none of which may happen to be at line center.

Separating Eqs. (27) and (29) into real and imaginary parts, one obtains

$$
0=-A_{r s, t h}-\int_{-\infty}^{\infty} P_{i s, t h}(V) \mathrm{d} V,
$$

$$
0=-A_{i s, t h}+\int_{-\infty}^{\infty} P_{r s, t h}(V) \mathrm{d} V
$$

$$
\begin{aligned}
& 0=P_{r s, t h}(V)-V P_{i s, t h}(V)-A_{i s, t h} D_{s, t h}(V), \\
& 0=P_{i s, t h}(V)+V P_{r s, t h}(V)+A_{r s, t h} D_{s, t h}(V) .
\end{aligned}
$$

One can combine Eqs. (32) and (33) to obtain

$$
\begin{gathered}
P_{r s, t h}(V)=\frac{\left(A_{i s, t h}-V A_{r s, t h}\right)}{\left(1+V^{2}\right)} D_{s, t h}(V), \\
P_{i s, t h}(V)=-\frac{\left(A_{r s, t h}+V A_{i s, t h}\right)}{\left(1+V^{2}\right)} D_{s, t h}(V),
\end{gathered}
$$

As mentioned above, at the lasing threshold the electric field and the polarization are infinitesimal. Therefore, the 
saturation terms in Eq. (23) can be omitted. In the absence of saturation, one can write Eqs. (23) and (24) as

$$
\begin{aligned}
& 0=\left(\lambda_{a s, t h}(V)-\lambda_{b s, t h}(V)\right)-h_{5} D_{s, t h}(V)-h_{6} M_{s, t h}(V), \\
& 0=\left(\lambda_{a s, t h}(V)+\lambda_{b s, t h}(V)\right)-h_{7} M_{s, t h}(V)-h_{8} D_{s, t h}(V),
\end{aligned}
$$

where the upper and lower level pump rates have been introduced using the formulas $\lambda_{d}(V)=\lambda_{a}(V)-\lambda_{b}(V)$ and $\lambda_{m}(V)=\lambda_{a}(V)+\lambda_{b}(V)$, and the additional $h$ coefficients are defined in Ref. 11.

Combining Eqs. (36) and (37), one can write

$$
\begin{aligned}
& D_{s, t h}(V)=\frac{1}{\gamma_{a} \gamma_{b}}\left[\left(\gamma_{b}-\gamma_{a b}\right) \lambda_{a s, t h}(V)-\gamma_{a} \lambda_{b s, t h}(V)\right], \\
& M_{s, t h}(V)=\frac{1}{\gamma_{a} \gamma_{b}}\left[\left(\gamma_{b}+\gamma_{a b}\right) \lambda_{a s, t h}(V)+\gamma_{a} \lambda_{b s, t h}(V)\right] .
\end{aligned}
$$

Now, combining Eqs. (30), (35), and (38) yields

$$
\begin{aligned}
0= & {\left[1-\frac{1}{\gamma_{a} \gamma_{b}} \int_{-\infty}^{\infty} \frac{\left(\gamma_{b}-\gamma_{a b}\right) \lambda_{a s, t h}(V)-\gamma_{a} \lambda_{b s, t h}(V)}{1+V^{2}} \mathrm{~d} V\right] A_{r s, t h} } \\
& -\frac{1}{\gamma_{a} \gamma_{b}}\left\{\int _ { - \infty } ^ { \infty } \frac { V } { 1 + V ^ { 2 } } \left[\left(\gamma_{b}-\gamma_{a b}\right) \lambda_{a s, t h}(V)\right.\right. \\
& \left.\left.-\gamma_{a} \lambda_{b s, t h}(V)\right] \mathrm{d} V\right\} A_{i s, t h} .
\end{aligned}
$$

In a similar fashion, Eqs. (31), (34), and (38) can be combined to obtain

$$
\begin{aligned}
0= & \frac{1}{\gamma_{a} \gamma_{b}}\left\{\int_{-\infty}^{\infty} \frac{V}{1+V^{2}}\left[\left(\gamma_{b}-\gamma_{a b}\right) \lambda_{a s, t h}(V)-\gamma_{a} \lambda_{b s, t h}(V)\right] \mathrm{d} V\right\} \\
& \times A_{r s, t h}+\left\{1-\frac{1}{\gamma_{a} \gamma_{b}}\right. \\
& \left.\times \int_{-\infty}^{\infty} \frac{\left(\gamma_{b}-\gamma_{a b}\right) \lambda_{a s, t h}(V)-\gamma_{a} \lambda_{b s, t h}(V)}{1+V^{2}} \mathrm{~d} V\right\} A_{i s, t h}
\end{aligned}
$$

Equations (40) and (41) form a set of linear homogeneous equations in parameters $A_{r s, t h}$ and $A_{i s, t h}$. A nontrivial solution exists if the determinant of the coefficient matrix of $A_{r s, t h}$ and $A_{i s, t h}$ equals zero. Therefore,

$$
\begin{aligned}
0= & {\left[1-\frac{1}{\gamma_{a} \gamma_{b}} \int_{-\infty}^{\infty} \frac{\left(\gamma_{b}-\gamma_{a b}\right) \lambda_{a s, t h}(V)-\gamma_{a} \lambda_{b s, t h}(V)}{1+V^{2}} \mathrm{~d} V\right]^{2} } \\
& -\left(\frac { 1 } { \gamma _ { a } \gamma _ { b } } \left\{\int _ { - \infty } ^ { \infty } \frac { V } { 1 + V ^ { 2 } } \left[\left(\gamma_{b}-\gamma_{a b}\right) \lambda_{a s, t h}(V)\right.\right.\right. \\
& \left.\left.\left.-\gamma_{a} \lambda_{b s, t h}(V)\right] \mathrm{d} V\right\}\right)^{2} .
\end{aligned}
$$

The second term of Eq. (42) is an odd function of the variable $V$ if Gaussian velocity distribution pumping functions are assumed, and therefore the integration vanishes. In this case Eq. (42) reduces to

$$
1=\frac{1}{\gamma_{a} \gamma_{b}} \int_{-\infty}^{\infty} \frac{\left(\gamma_{b}-\gamma_{a b}\right) \lambda_{a s, t h}(V)-\gamma_{a} \lambda_{b s, t h}(V)}{1+V^{2}} \mathrm{~d} V .
$$

Next, assume Gaussian pumping functions of the forms

$$
\begin{aligned}
& \lambda_{a}(V, \eta, \tau)=\lambda_{a}(V)=\frac{\varepsilon L_{a}}{\sqrt{\pi}} \exp \left(-\varepsilon^{2} V^{2}\right), \\
& \lambda_{b}(V, \eta, \tau)=\lambda_{b}(V)=\frac{\varepsilon L_{b}}{\sqrt{\pi}} \exp \left(-\varepsilon^{2} V^{2}\right) .
\end{aligned}
$$

Then substitute these functions into Eq. (43) and rewrite it as

$$
1=\frac{\varepsilon}{\sqrt{\pi}} \int_{-\infty}^{\infty}\left[\frac{\left(\gamma_{b}-\gamma_{a b}\right)}{\gamma_{a} \gamma_{b}} L_{a}-\frac{\gamma_{a}}{\gamma_{a} \gamma_{b}} L_{b}\right] \frac{\exp \left(-\varepsilon^{2} V^{2}\right)}{\left(1+V^{2}\right)} \mathrm{d} V
$$

$$
\begin{aligned}
& =\frac{\varepsilon}{\sqrt{\pi}} \int_{-\infty}^{\infty}\left[\left(1-\frac{\gamma_{a b}}{\gamma_{b}}\right) \frac{L_{a}}{\gamma_{a}}-\frac{L_{b}}{\gamma_{b}}\right] \frac{\exp \left(-\varepsilon^{2} V^{2}\right)}{\left(1+V^{2}\right)} \mathrm{d} V \\
& =\frac{\varepsilon}{\sqrt{\pi}} L_{a}\left[\left(1-\frac{\gamma_{a b}}{\gamma_{b}}\right) \frac{1}{\gamma_{a}}-\frac{q}{\gamma_{b}}\right] \int_{-\infty}^{\infty} \frac{\exp \left(-\varepsilon^{2} V^{2}\right)}{\left(1+V^{2}\right)} \mathrm{d} V,
\end{aligned}
$$

where $q=L_{b} / L_{a}$. Equation (48) can be written in terms of the threshold parameter $\mathfrak{R}$ and the threshold pumping parameter $L_{a, t h}$ as

$$
\begin{aligned}
L_{a}= & \Re L_{a, t h} \\
= & \Re \frac{\sqrt{\pi}}{\varepsilon}\left[\left\{\left(1-\frac{\gamma_{a b}}{\gamma_{b}}\right) \frac{1}{\gamma_{a}}-\frac{q}{\gamma_{b}}\right\}\right. \\
& \left.\times \int_{-\infty}^{\infty} \frac{\exp \left(-\varepsilon^{2} V^{2}\right)}{\left(1+V^{2}\right)} \mathrm{d} V\right]^{-1} .
\end{aligned}
$$

Equation (50) is used in our numerical calculations to set the pump rates in terms of the threshold parameter $\mathfrak{R}$.

Prior to computing the time-dependent solutions of the dynamical Eqs. (20) and (22)-(24), it is necessary to choose initial values of certain dynamical variables. A useful approach is based on the method of loss switching ( $Q$ switching). It is used experimentally to generate short pulses and it also encourages the initiation of laser instabilities. Conceptually, the laser medium is assumed to be pumped to some initial distribution of population that corresponds to a specific value of the threshold parameter, while the electric field is held at zero. A small initial value of electric field is then introduced to start the computations. Thus at the outset the only nonzero dependent variables are the Gaussian pump rates, population difference, and the population sum in Eqs. (22)-(24). 

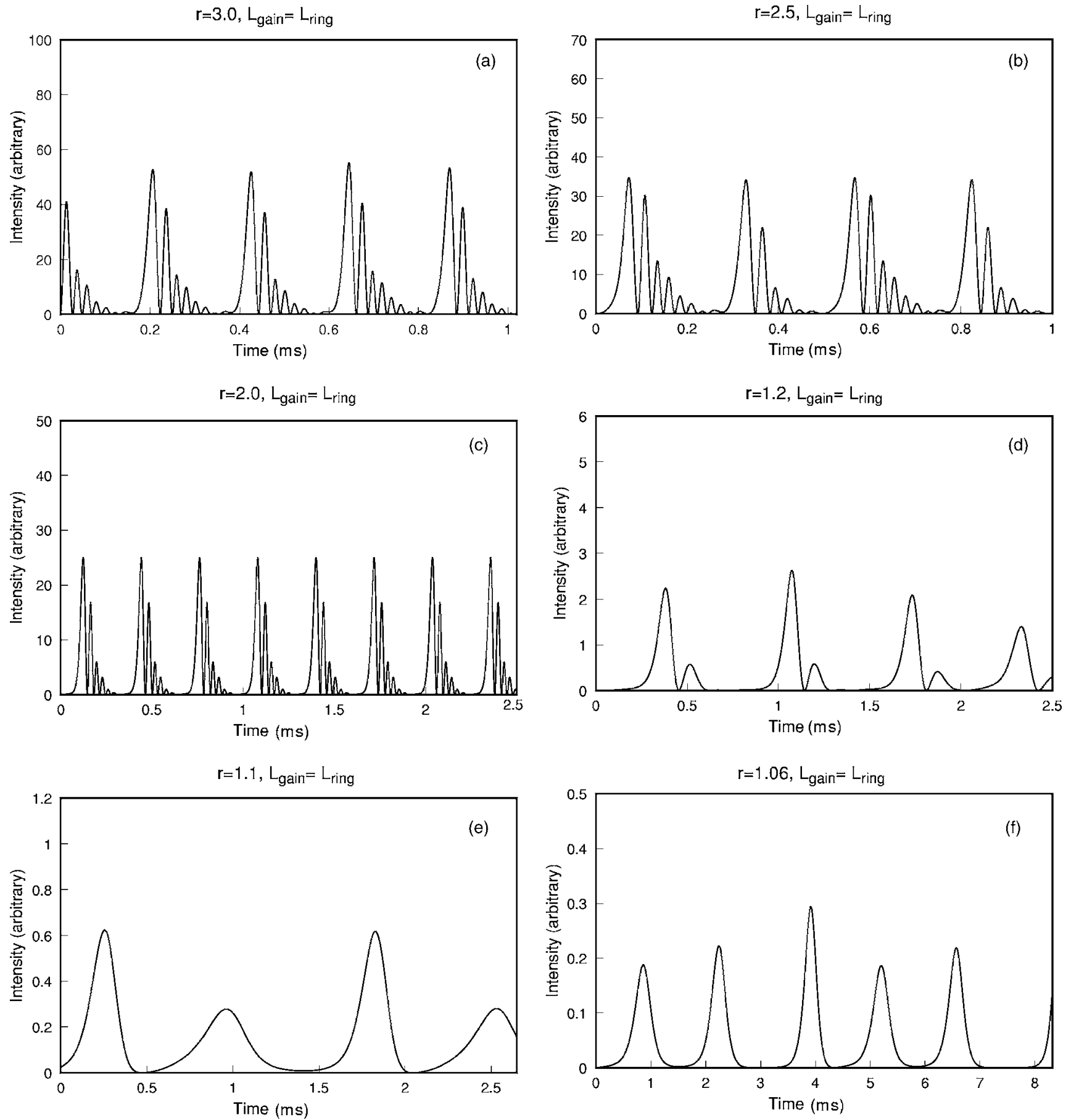

Fig. 1. Theoretical spontaneous-pulsation waveforms for a single-mode xenon laser with line-center tuning and uniformly distributed loss. The threshold parameter values are $\mathfrak{R}=$ (a) 3.0, (b) 2.5, (c) 2.0, (d) 1.2, (e) 1.1, (f) 1.06. These are the same conditions as those in Fig. 2 of Ref. 5.

Combining Eqs. (38), (39), (44), and (45), one obtains the values of $D_{\text {init }}(V)$ and $M_{\text {init }}(V)$ for prepumped material as

$$
\begin{aligned}
D_{\text {init }}(V)= & \frac{1}{\gamma_{a} \gamma_{b}}\left[\left(\gamma_{b}-\gamma_{a b}\right) \frac{\varepsilon L_{a}}{\sqrt{\pi}} \exp \left(-\varepsilon^{2} V^{2}\right)\right. \\
& \left.-\gamma_{a} \frac{\varepsilon L_{b}}{\sqrt{\pi}} \exp \left(-\varepsilon^{2} V^{2}\right)\right]
\end{aligned}
$$

$$
\begin{gathered}
=\frac{\varepsilon}{\sqrt{\pi}}\left[\left(1-\frac{\gamma_{a b}}{\gamma_{b}}\right) \frac{L_{a}}{\gamma_{a}}-\frac{L_{b}}{\gamma_{b}}\right] \exp \left(-\varepsilon^{2} V^{2}\right), \\
M_{\text {init }}(V)=\frac{1}{\gamma_{a} \gamma_{b}}\left[\left(\gamma_{b}+\gamma_{a b}\right) \frac{\varepsilon L_{a}}{\sqrt{\pi}} \exp \left(-\varepsilon^{2} V^{2}\right)\right. \\
\left.+\gamma_{a} \frac{\varepsilon L_{b}}{\sqrt{\pi}} \exp \left(-\varepsilon^{2} V^{2}\right)\right]
\end{gathered}
$$



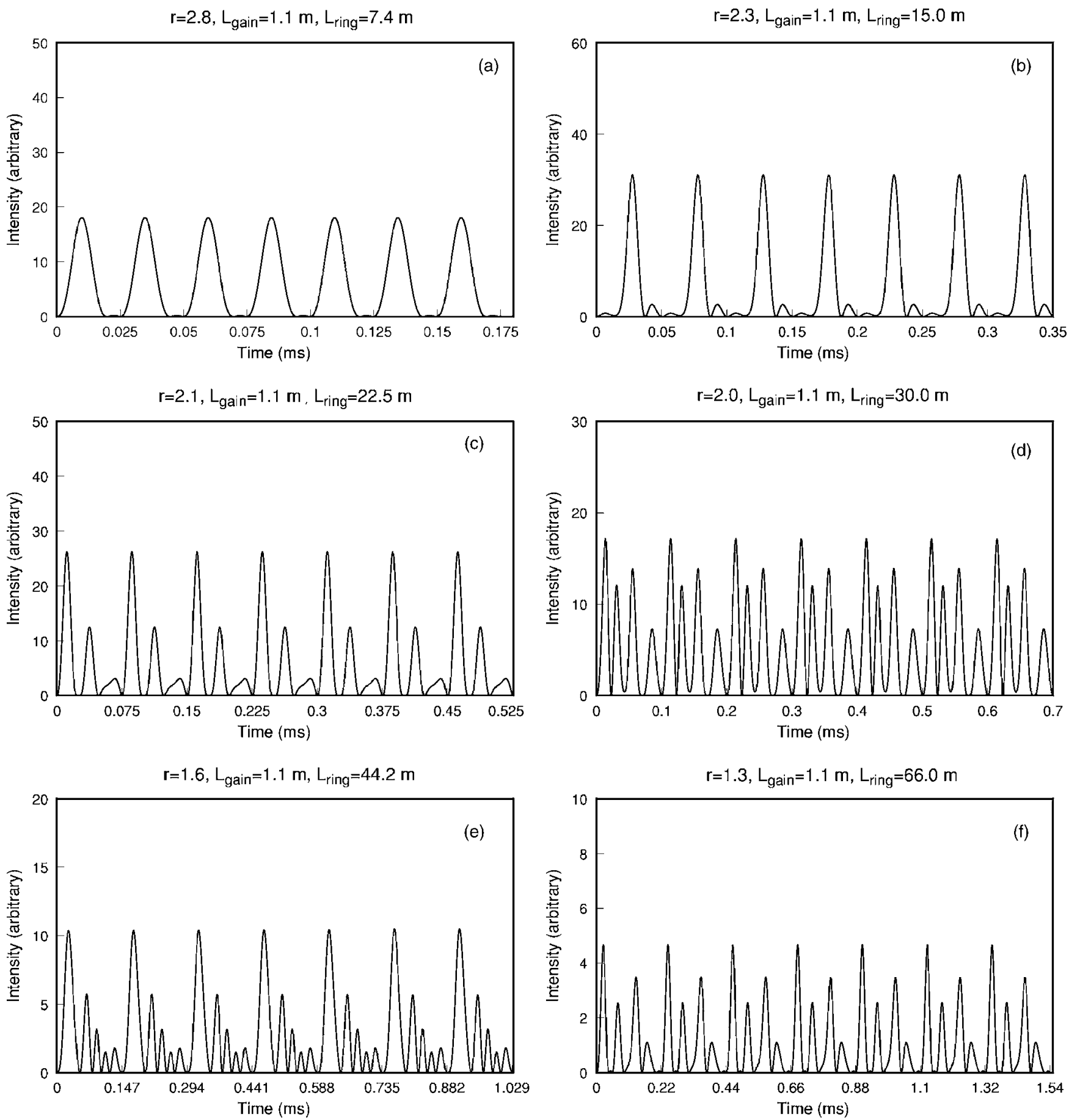

Fig. 2. Theoretical self-locking waveform for a long-cavity xenon laser. The length of the gain medium is $1.1 \mathrm{~m}$. The cavity lengths and the threshold parameter values are: (a) $L_{\text {cavity }}=7.4 \mathrm{~m}, \mathfrak{R}=2.8$; (b) $L_{\text {cavity }}=15 \mathrm{~m}, \mathfrak{R}=2.3$; (c) $L_{\text {cavity }}=22.5 \mathrm{~m}$; $\mathfrak{R}=2.1$; (d) $L_{\text {cavity }}=30 \mathrm{~m}$; $\mathfrak{R}$ $=2.0$; (e) $L_{\text {cavity }}=44.2 \mathrm{~m}, \mathfrak{R}=1.6$; (f) $L_{\text {cavity }}=66 \mathrm{~m}, \mathfrak{R}=1.3$.

$$
=\frac{\varepsilon}{\sqrt{\pi}}\left[\left(1+\frac{\gamma_{a b}}{\gamma_{b}}\right) \frac{L_{a}}{\gamma_{a}}+\frac{L_{b}}{\gamma_{b}}\right] \exp \left(-\varepsilon^{2} V^{2}\right) .
$$

These prepumped values of population parameters are used at the start of numerical computations. In addition, the other dependent variables in Eqs. (20) and (22)-(24) are initially set to zero except for the small starting value of the electric field amplitude.

\section{RESULTS}

In the preceding sections a mathematical model was developed that, in principle, describes the time and space dependences of the electromagnetic fields propagating around a ring-laser cavity. However, in their present forms, as in Eqs. (20) and (22)-(24), the semiclassical laser equations are not directly suitable for numerical computation of the dynamical behavior of the laser. It is necessary to apply a discretization method to the laser 

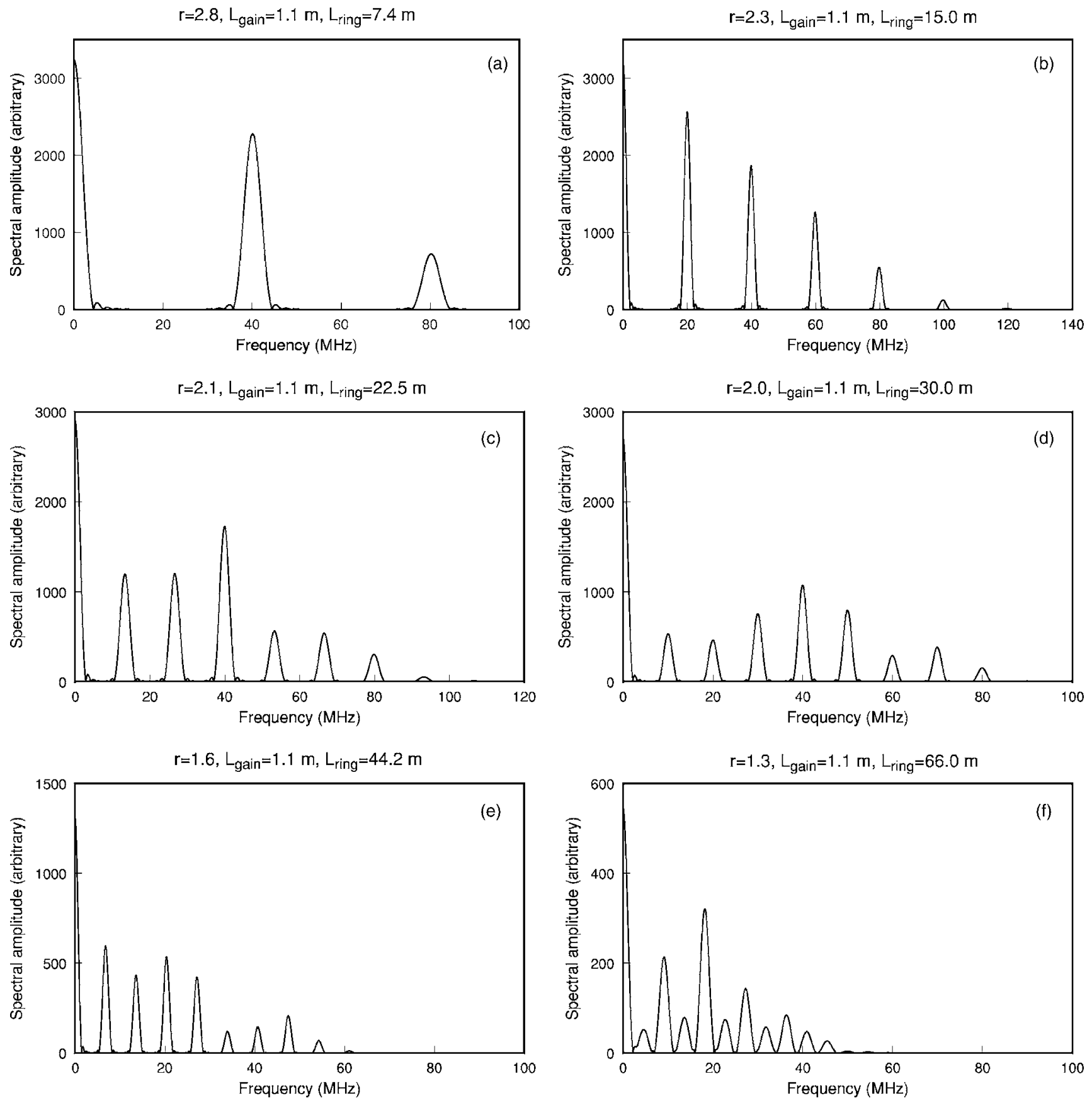

Fig. 3. Frequency spectra of the theoretical self-locking waveforms in Fig. 2. The length of the gain medium is $1.1 \mathrm{~m}$. The cavity lengths and the threshold parameter values are, respectively, the same as in Fig. 2.

equations and obtain the final set of equations that can be numerically solved on a computer.

We adopt the discretization scheme that was used in Ref. 12, which is similar to that employed by Risken and Nummedal. ${ }^{2}$ The first-order space and time derivatives of the laser dynamical variables are discretized at every two-dimensional (spatial and temporal) grid point $(m, n)$ according to the Taylor expansion formulas

$$
\frac{\partial X(m, n)}{\partial \eta}=\frac{X(m, n)-X(m-1, n)}{\Delta \eta}+\frac{1}{2} \Delta \eta \frac{\partial^{2} X(m, n)}{\partial \eta^{2}},
$$

$$
\frac{\partial X(m, n)}{\partial \tau}=\frac{X(m, n+1)-X(m, n)}{\Delta \tau}-\frac{1}{2} \Delta \tau \frac{\partial^{2} X(m, n)}{\partial \tau^{2}},
$$

where the second-order space and time derivatives of the dynamical variables will be obtained directly from the laser equations. Equation (20) suggests that the spatial step size $\Delta \eta$ and the temporal step size $\Delta \tau$ are related by

$$
\Delta \eta=v_{p} \frac{l}{L} \Delta \tau .
$$



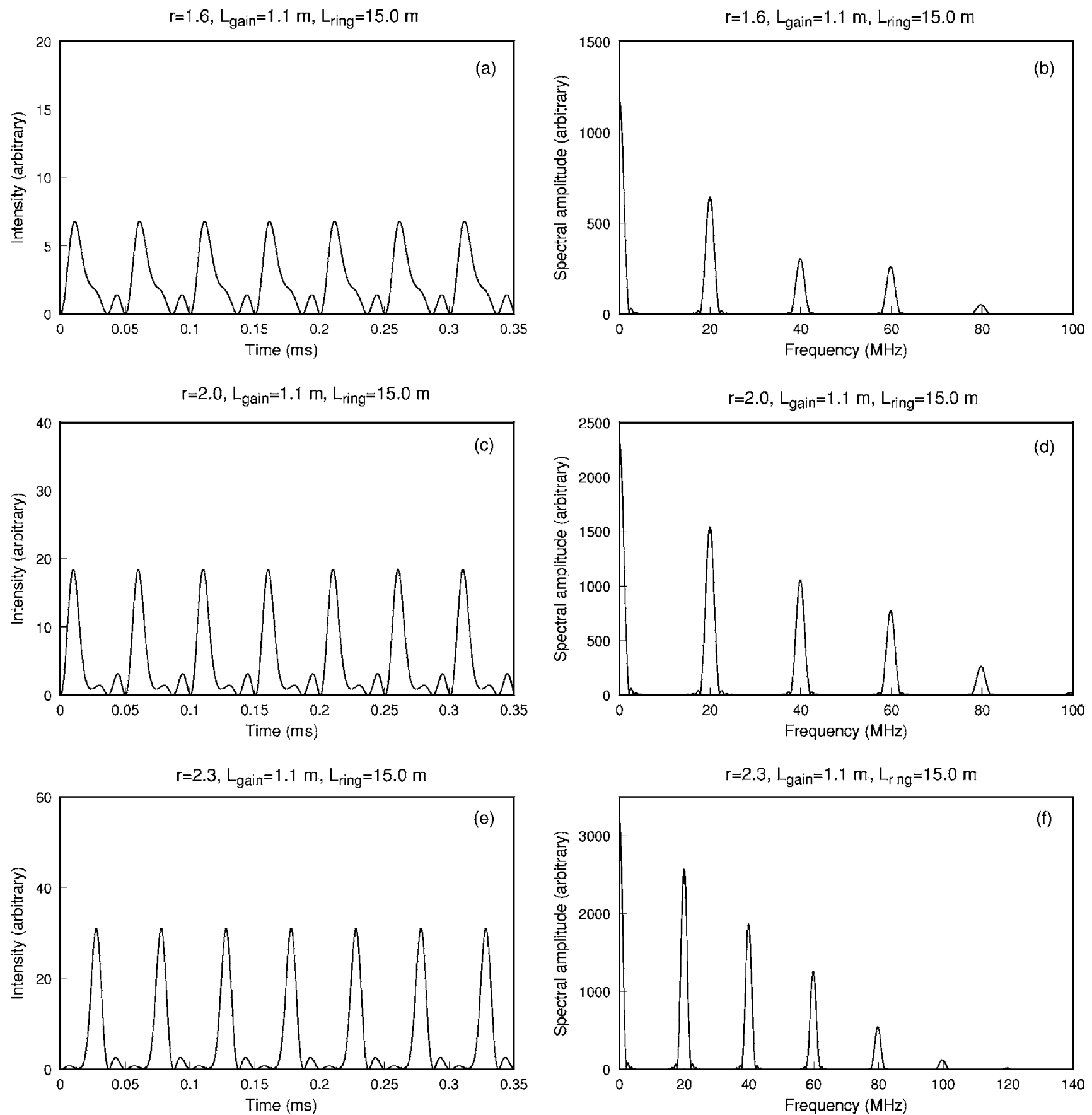

Fig. 4. Theoretical self-locking waveforms and the corresponding frequency spectra for a long-cavity xenon laser. The gain medium and cavity lengths are 1.1 meters and 15 meters, respectively. The threshold parameter values are: $\Re=$ (a) 1.6 , (b) 2.0 , (c) 2.3 .

The discretized set of laser equations can be obtained by substituting Eqs. (20) and (22)-(24) into Eqs. (55) and (56). This process is straightforward but tedious, and the results are omitted here. Further information on the derivations is available in Ref. 13 . To establish the validity of the derived model, we apply the set of discretized dynamical laser equations to the specific case of a laser configuration where both the active medium and the resonant cavity have identical length. In particular, we are first interested in the comparisons between the previously obtained theoretical calculations of spontaneous coherent pulsations in xenon lasers from Ref. 8 and those from our model. The results of calculations for laser operating con- ditions and parameter values as investigated in Ref. 8 using our current model are shown in Fig. 1. Comparison of these results to Fig. 2 in Ref. 8 shows good agreement.

Our main objective is to investigate the spontaneous, coherent, self-mode-locking behaviors in long-cavity, unidirectional ring-laser oscillators. Tarroja et al. ${ }^{1}$ have conducted a series of experiments demonstrating spontaneous, coherent self-mode-locking in a long-cavity xenon laser. Results of the preliminary theoretical calculations shown in Ref. 1 could not explain additional dynamical behaviors such as the increased number of pulses per cavity round trip as the cavity length is increased. The following more detailed discussions of our results compare 

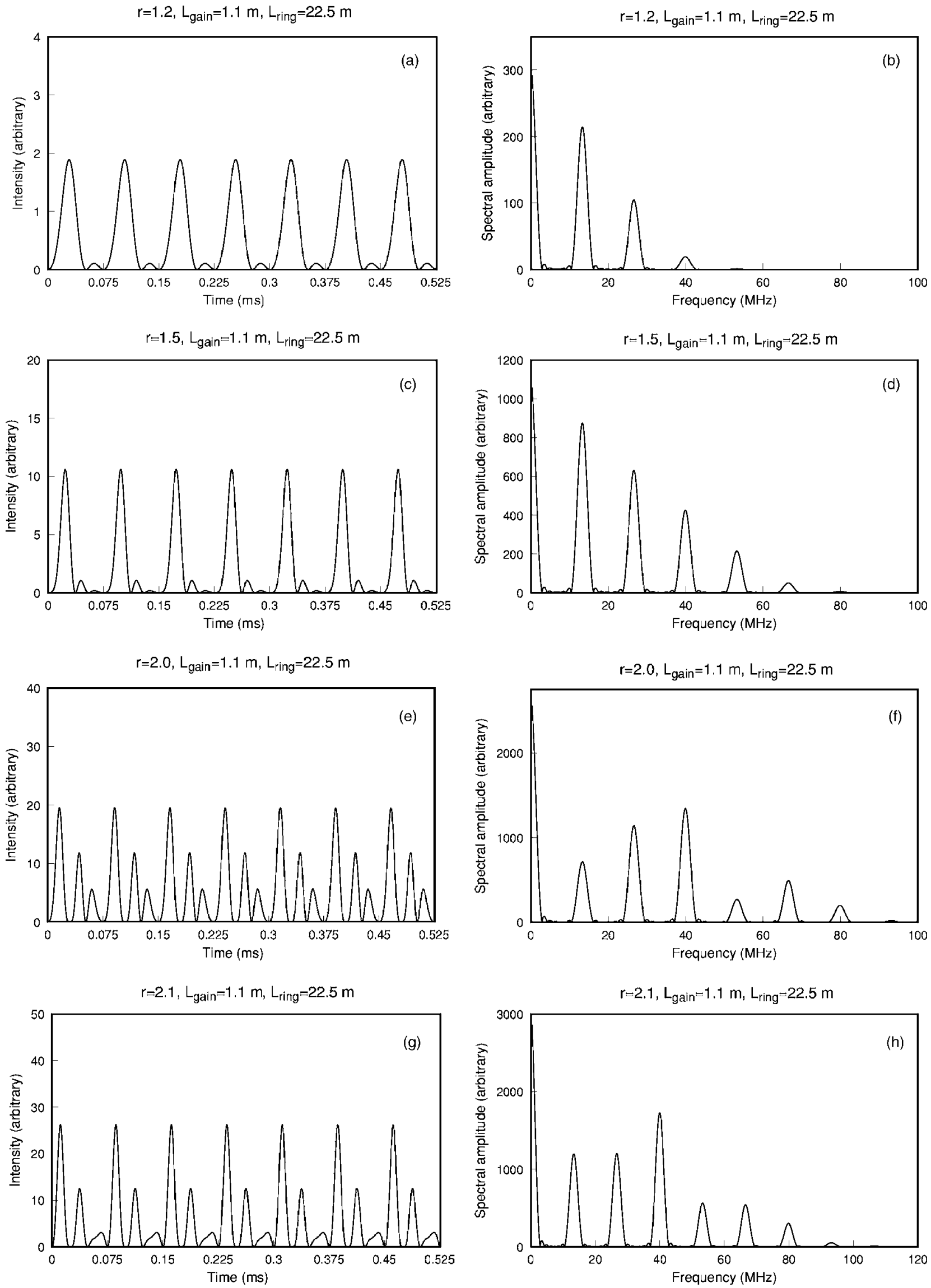

Fig. 5. Theoretical self-locking waveforms and the corresponding frequency spectra for a long-cavity xenon laser. The gain medium and cavity lengths are 1.1 meters and 22.5 meters, respectively. The threshold parameter values are: $\mathfrak{R}=$ (a) 1.2 , (b) 1.5 , (c) 2.0 , (d) 2.1 . 
specific theoretical predictions of the model presented here to the corresponding experimental results for the low-pressure xenon laser presented in Ref. 1. Areas of comparison include the time-domain intensity waveforms and homodyne spectra as functions of cavity length and threshold parameter.

The results of calculations using the current model in the case of different gain media and cavity lengths are shown in Figs. 2-5. For these calculations, we adopted the same values of material parameters and cavity lifetime as used in Ref. 1. The results show that as the cavity length is increased, a higher number of laser pulses in a cavity round-trip time can be observed. In interpreting standing-wave laser data with a ring-laser model, the round-trip ring length should equal twice the single-pass length of the standing-wave laser. This ensures that the laser atoms interact with laser fields having the correct pulsation rates and mode frequency spacings. For an extended cavity length of $15.0 \mathrm{~m}$, as represented in Fig. 2(b), a single pulse is typically found to circulate about the cavity with only a minor level of structure depending on the pump level. This theoretical ring-laser result may be seen to correspond closely with the experimental result for a standing-wave laser of length $7.4 \mathrm{~m}$ shown in Fig. 2(a) of Ref. 1. With larger cavity lengths, increasing numbers of pulses circulate around the cavity. The theoretical plot for a ring-laser cavity length of $44.2 \mathrm{~m}$ shown in Fig. 2(e) corresponds approximately to the experimental time exposure for a standing-wave laser of length $22.8 \mathrm{~m}$ shown in Fig. 2(b) of Ref. 1.

The homodyne spectra of the laser output corresponding to the time domain plots in Fig. 2 are shown in Fig. 3. The frequency spectrum for a $44.2 \mathrm{~m}$ standing-wave laser, for example, shown in Fig. 3 corresponds approximately with the experimental spectrum for a standing-wave laser of length $22.5 \mathrm{~m}$ shown in Fig. 7(b) of Ref. 1. In addition, at a fixed cavity length, the pulsation characteristics become less complex as the laser operates closer to the lasing threshold. This is evident from the theoretical plots of Figs. 4 and 5 and from the experimental results of Figs. 5 and 6 in Ref. 1. The ringing feature of the fundamental pulse is also apparent in many of the theoretical and experimental plots.

As emphasized above, the model reported here provides good agreement with many features of the experimental data for an extended-cavity, spontaneously mode-locked xenon laser oscillator. Using only previously established parameter values, theoretical pulsation waveforms and homodyne spectra have many features in common with the reported experimental results. One perceptible discrepancy, however, concerns the regularity of the pulsation waveforms. For low to moderate values of the threshold parameter for a given cavity length, the individual pulsations or pulsation bursts in the theoretical results are more nearly periodic than the experimental results. To emphasize the nonperiodicity of the experiments, the waveforms in Ref. 1 were presented in both single-shot [Figs. 3(a), 3(b), and 4] and multitrace [Figs. 2 and 5, 6] form. The fuzziness of the pulses in the multitrace recordings reflects the partial lack of periodicity in the singleshot results.

The probable reason for the discrepancy that has just been noted is that our theoretical model is based on a ring-laser configuration, while the available experimental data were obtained using standing-wave lasers. The analogous effect has been noted previously with singlemode lasers. Spontaneous coherent pulsations in singlemode xenon lasers have been modeled for both ring and standing-wave configurations. As discussed previously, the calculated single-mode intensity waveforms in the standing-wave case are "much less regular than in the corresponding ring laser." ${ }^{0}$ That irregularity was reflected similarly in a fuzziness in the multitrace experimental recordings.

The greater complexity of the standing-wave laser behavior is related in general to the necessarily greater complexity of the underlying mathematical model. In the simplest distributed-loss ring-laser model the active laser atoms or molecules all interact with essentially the same traveling-wave electromagnetic field. By contrast, in the most basic standing-wave laser model the atoms interact with the interference pattern of the right and left traveling components that constitute the field. Thus, the field that the individual moving atoms experience is a more involved function of space and time, and the corresponding laser model requires a more complicated nonlinear set of differential equations for its mathematical representation. A more complicated dynamical model usually implies a greater richness in the kinds of periodic or chaotic solutions, and that is the case for spontaneously pulsing lasers.

\section{CONCLUSION}

A theoretical model has been presented for spontaneous, coherent self-mode-locking in long-cavity, mixedbroadened laser oscillators. The starting point for this analysis is the same semiclassical formalism employed in previous instability studies of single-mode ring and standing-wave lasers. The more complicated time and space variations that occur in a multimode laser are handled by means of a discretization of the laser equations. This puts the equations into a form that is appropriate for efficient numerical computation.

The multimode model described here includes the single-mode laser as a special case. This single-mode limit has been compared with already known single-mode results as a check on the model. More general multimode calculations using xenon laser parameters have then been compared with previously published data on spontaneous mode locking. In the theory and experiments the number of pulses per round trip is found to increase with cavity length and pumping rate. Good agreement has been obtained in both the time and frequency domains, and the model described here provides an accurate representation of the spontaneous, mode-locking pulsation data obtained for mixed-broadened laser oscillators.

Corresponding author L. Casperson's e-mail address is lcaspers@uncc.edu.

\footnotetext{
*Present address, Laser Technology Group, Maxim Integrated Products, 7250 Northwest Evergreen Parkway, Hillsboro, Oregon 97124, USA.
} 
${ }^{\dagger}$ Present address, Department of Electrical and Computer Engineering and Center for Optoelectronics and Optical Communications of the University of North Carolina at Charlotte, 9201 University City Boulevard, Charlotte, North Carolina 28223, USA.

\section{REFERENCES}

1. M. F. H. Tarroja, M. Sharafi, and L. W. Casperson, "Spontaneous mode locking in long-cavity lasers," J. Opt. Soc. Am. B 6, 1564-1573 (1989).

2. H. Risken and K. Nummedal, "Self-pulsing in lasers," J. Appl. Phys. 39, 4662-4672 (1968).

3. H. Haken, "Analogy between higher instabilities in fluids and lasers," Phys. Lett. A 53, 77-78 (1975).

4. U. Hubner, N. B. Abraham, and C. O. Weiss, "Dimensions and entropies of chaotic intensity pulsations in a singlemode far-infrared $\mathrm{NH}_{3}$ laser," Phys. Rev. A 40, 6354-6365 (1989).

5. T. Voigt, M. O. Lenz, F. Mitschke, E. Roldan, and G. J. De Valcarcel, "Experimental investigation of Risken-Nummedal-Graham-Haken laser instability in fiber ring lasers," Appl. Phys. B: Lasers Opt. 79, 175-183 (2004).

6. H. H. Kim and H. Marantz, "Continuous self-mode locking of infrared gas lasers," IEEE J. Quantum Electron. QE-6, 749-750 (1970).

7. L. W. Casperson and A. Yariv, "Pulse propagation in a high-gain medium,” Phys. Rev. Lett. 26, 293-295 (1971).

8. L. W. Casperson, "Spontaneous coherent pulsations in ringlaser oscillators," J. Opt. Soc. Am. B 2, 62-72 (1985).

9. E. I. Gordon, A. D. White, and J. D. Rigden, "Gain saturation at 3.39 microns in the $\mathrm{He}-\mathrm{Ne}$ maser," in Proceedings of the Symposium on Optical Masers, Polytechnic Institute of Brooklyn, New York, 309-317 (Wiley, 1964).

10. L. W. Casperson, "Spontaneous coherent pulsations in standing-wave laser oscillators," J. Opt. Soc. Am. B 5, 958-969 (1988).

11. L. W. Casperson and M. F. H. Tarroja, "Spontaneous coherent pulsations in standing-wave laser oscillators: simplified models," J. Opt. Soc. Am. B 8, 250-261 (1991).

12. L. M. Narducci, J. R. Tredicce, L. A. Lugiato, N. B. Abraham, and D. K. Bandy, "Mode-mode competition and unstable behavior in a homogeneously broadened ring laser," Phys. Rev. A 33, 1842-1854 (1986).

13. P. Chenkosol, "Spontaneous pulsations in laser oscillators: effects of spatial field distributions; self-mode-locking dynamics; pulsations in $3.39 \mu \mathrm{m} \mathrm{He}-\mathrm{Ne}$ standing-wave lasers," Ph.D. dissertation (Portland State University, Portland, Oregon, USA, 2003), Appendix A, pp. 196-200. 